\title{
Quality Assessment For Stereoscopic Images With JPEG Compression Errors
}

\author{
Kenny H.B. Voo and David B.L. Bong \\ Faculty of Engineering Universiti Malaysia Sarawak, 94300, Kota Samarahan, Malaysia
}

\begin{abstract}
In this paper a new quality assessment method for stereoscopic image with compression distortion is proposed. This method is designed based on hybrid of $2 \mathrm{D}$ image quality metrics and stereoscopic depth map information. The proposed metric is tested on LIVE 3D image database with JPEG and JPEG2000 (JP2K) errors. As a result, this metric has shown an improved performance in assessing perceived quality for stereoscopic images.
\end{abstract}

\section{INTRODUCTION}

Rapid development in stereoscopic/3D imaging has caused many researchers to advance their researches in this field. Moreover, stereoscopic imaging has been widely used in different areas. Thus, in assessing quality of stereoscopic images we believe that the depth map for the corresponding images plays an essential role. Hence in this work, we had included depth element in assessing the quality of stereoscopic images.

\section{NEW SSIM WITH DEPTH ELEMENT}

In [1], Moorthy et al. mentioned that the sensibility of stereo vision is caused by the difference in angle coordination between both eyes. This difference of viewpoint is known as the depth of the stereo image. Depth information is illustrated in Figure 1.

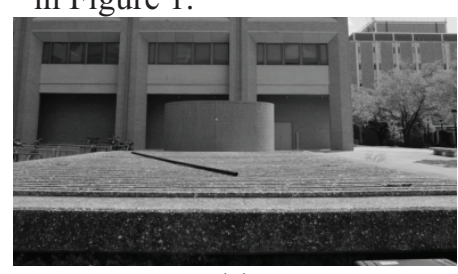

(a)

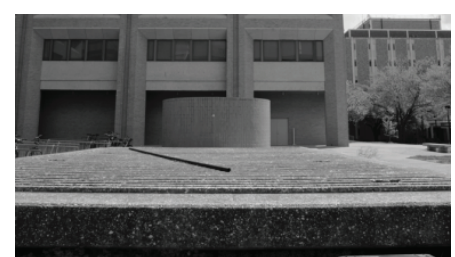

(b)

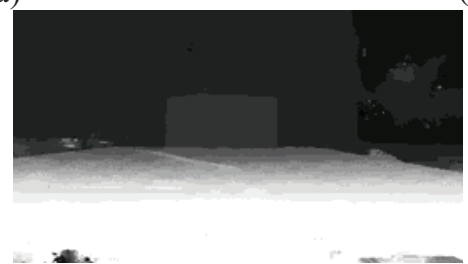

(c)

Figure 1: (a) Left reference image (b) Right reference image (c) Corresponding depth map

Depth map in Figure 1(c) shows that the color represented in a depth map consists of only gray scale, which the section with darker color represents the surface is further from a point of

The authors would like to acknowledge Ministry of Education Malaysia, for the provision of research grant: FRGS/TK03(01)/1063/2013(09) and Faculty of Engineering, Universiti Malaysia Sarawak for the provision of research facilities. view where else, section with lighter color represents the surface is nearer from a point of view. For our work, Fast Cost Volume Filtering method [2] has been chosen in generating depth maps from stereoscopic images.

Structural Similarity Index also known as SSIM [3], is one of the popular 2D quality metrics which was proposed by Wang et al. It is an efficient quality assessment method as compared to subjective evaluation [4], and is able to provide quality scores which are close to human perception. SSIM assesses images quality by measuring the similarity between two corresponding images, where both images are defined as $x$ and $y$. Mathematical, SSIM metric can be defined as [5].

$$
\operatorname{SSIM}(x, y)=\frac{\left(2 \mu_{x} \mu_{y}+C_{1}\right)\left(2 \sigma_{x y}+C_{2}\right)}{\left(\mu_{x}^{2}+\mu_{y}^{2}+C_{1}\right)\left(\sigma_{x}^{2}+\sigma_{y}^{2}+C_{2}\right)}
$$

For our proposed metric, we have modified SSIM and introduced STEREO-SSIM which can be modeled as:

$$
\begin{aligned}
& \operatorname{STEREO}-\operatorname{SSIM}(x, y)= \\
& \frac{\left(2 \mu_{x} \mu_{y}+C_{1}\right)\left(2 \sigma_{x y}+C_{2}\right)\left(\alpha \sigma^{\beta}{ }_{x_{1} y_{1}}+C_{2}\right)}{\left(\mu_{x}^{2}+\mu_{y}^{2}+C_{1}\right)\left(\sigma_{x}^{2}+\sigma_{y}^{2}+C_{2}\right)\left(X \sigma_{x_{1}}^{2^{\gamma}}+Y \sigma_{y_{1}}^{2^{\delta}}+C_{2}\right)}
\end{aligned}
$$

In equation (2), the proposed metric has been modified from the SSIM model by including $x_{1}$ and $y_{1}$ in the equation which is the depth information. $x_{1}$ is the depth map for the reference stereoscopic images, whereas $y_{l}$ is the depth map for distortion stereoscopic images. Parameters X, Y, $\alpha, \beta, \gamma$ and $\delta$ are also constant positive values, which are needed to adapt the significant values of the depth component.

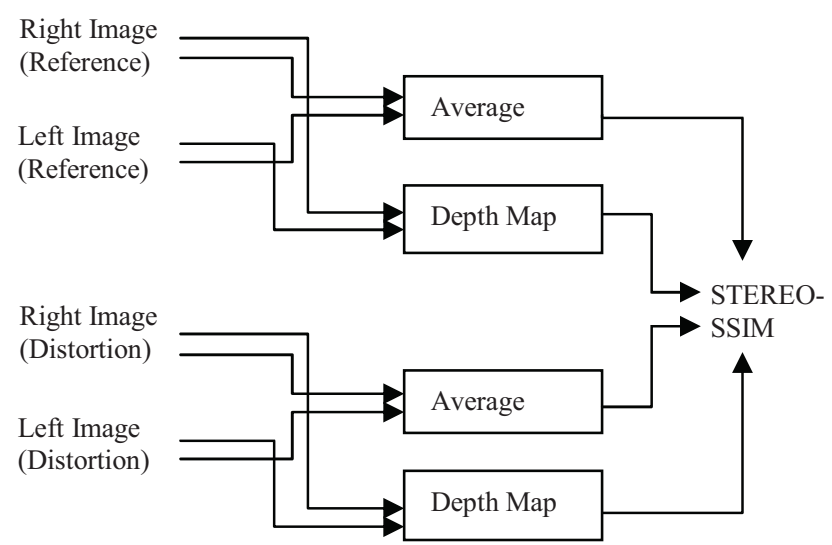

Figure 2: Proposed method in assessing the stereoscopic quality images using STEREO-SSIM

Figure 2 shows the approach of STEREO-SSIM. First, both left and right original images are averaged. Likewise, the averaging process is also applied to the left and right distorted images. Then, with the obtained depth maps for the 